\section{Características das gestações, partos e recém-nascidos da região metropolitana de Aracaju, Sergipe, Brasil}

\section{Characteristics of pregnancies, deliveries and newborns in the Metropolitan Region of Aracaju, State of Sergipe, Brazil}

\section{Abstract}

Objectives: to describe the characteristics of pregnancies, deliveries and the status of newborns at birth in the Metropolitan Area of Aracaju. The main demographic and socioeconomic characteristics and details of the medical care given to the population under study are presented.

Methods: a cross-sectional study was carried out of all deliveries in the maternity hospitals of Aracaju, in the State of Sergipe, Brazil, from March-July 2005. All mothers who gave birth to a single live baby were interviewed regarding their background, reproductive history and sexual life. Hospital records, including the babies weight, height and cephalic perimeter, were obtained and all information transferred to a standardised questionnaire. Data were compared to equivalent findings from other studies in Brazil.

Results: there were 4746 single live births during the study period in Aracaju. The majority of mothers (69\%) were considered poor (income $<3$ minimum wages). Adolescent mothers comprised $20.6 \%$ of the population. The figures for low birth-weight (7.2\%) and prematurity (7.7\%) were similar. Almost all mothers (98.3\%) received prenatal care, $76.0 \%$ from public services - the Sistema Único de Saúde (SUS) - which financed most deliveries. Caesarean sections accounted for $31.6 \%$ of all deliveries.

Conclusions: deliveries in Aracaju's showed good performance with a lower rate for Caesarean sections, prematurity, and low birth-weight than Ribeirão Preto, São Luis and Pelotas. Perinatal health planning may benefit from this kind of study.

Key words Child health, Mother's health, Birth, Delivery, Newborn
Ricardo Queiroz Gurgel 1

Alzira Maria D'Avila Guimarães Nery 2

Maria Luíza Dória Almeida 3

Eleonora Ribeiro Ramos Oliveira 4

Danilo Dantas Freire Lima 5

Heloísa Bettiol 6

Marco Antonio Barbieri 7

1,3-5 Departamento de Medicina. Universidade Federal de Sergipe. Rua Claudio Batista s.n. Bairro Sanatório. Aracaju, SE, Brasil. CEP: 49.000-100. E-mail: ricardoqg@infonet.com.br 2 Departamento de Enfermagem. Universidade Federal de Sergipe. Aracaju, SE, Brasil.

6,7 Departamento de Puericultura e Pediatria. Faculdade de Medicina de Ribeirão Preto. Universidade de São Paulo. Ribeirão Preto, SP, Brasil.

\section{Resumo}

Objetivos: descrever as características das gestações, partos e nascimentos da região metropolitana de Aracaju. As principais características demográficas e socioeconômicas e de atenção à saúde da população foram avaliadas.

Métodos: estudo transversal dos nascimentos ocorridos entre março e julho de 2005, procedentes da região metropolitana de Aracaju, Sergipe, Brasil. Todas as mães que tiveram parto único nascido vivo foram entrevistadas através de um questionário estruturado, com informações sobre as condições demográficas, socioeconômicas, história sexual/reprodutiva. Registros do peso, comprimento e perímetro cefálico das crianças foram transferidos para o questionário. Foi feita comparação com outros estudos brasileiros de caracteristicas semelhantes.

Resultados: foram analisados 4746 nascimentos de parto único de Aracaju, com 69\% das mães referindo renda inferior a três salários mínimos. As mães adolescentes representaram 20,6\% da amostra. Entre todos os nascimentos, 7,7\% tiveram duração inferior a 37 semanas, percentual semelhante ao de baixo peso ao nascer (7,2\%). Prevaleceu a assistência pré-natal oferecida pelo Sistema Único de Saúde (SUS) (76,0\%), que custeou $85,2 \%$ dos partos. Destes, 31,6\% foram cesáreas.

Conclusões: o estudo mostrou menores percentuais de partos cesária, prematuridade e baixo peso ao nascer que os encontrados em Ribeirão Preto, São Luis e Pelotas. Estudos como este podem ser muito úteis ao planejamento de saúde perinatal.

Palavras-chave Saúde infantil, Saúde materna, Nascimento, Parto, Recém-nascido 


\section{Introdução}

Os estudos de indicadores sociais e de saúde na população materna e infantil buscam o conhecimento mais apurado de alguns parâmetros mensuráveis das condições de vida e saúde da população. ${ }^{1}$

No Brasil, nas últimas décadas, alguns estudos epidemiológicos de grande porte, a exemplo, estudos de coorte, vêm sendo desenvolvidos. ${ }^{1-5}$ Entretanto, no Estado de Sergipe, poucas investigações na área materna e infantil têm sido realizadas, e nenhuma das pesquisas teve a amplitude de um estudo que contemplasse uma amostra tão representativa da população de nascimentos da região metropolitana de Aracaju.

O acompanhamento da evolução de indicadores de saúde materno infantil é essencial para avaliar o impacto de mudanças sociais e econômicas, como também os avanços ou retrocessos na disponibilidade e qualidade dos serviços de saúde. A monitorização deve incluir estudos de tendências em indicadores de saúde baseados em estatísticas de rotinas e sistemas de vigilância. Esses, no entanto, podem ser afetados por problemas como o sub-registro, cobertura incompleta de informações e a falta de padronização de informações. 6 Dessa forma, faz-se imprescindível a realização de pesquisas de base populacional, com desenhos metodológicos delineados adequadamente, para a mensuração e análise dos eventos em estudo.

Com base nessas premissas, os principais objetivos desse estudo foram: descrever as características das gestações, partos e nascimentos da região metropolitana de Aracaju em relação aos aspectos demográficos, econômicos, sociais e de atenção à saúde da população pesquisada. Dessa forma será possível avaliar a utilização de serviços de saúde; analisar o comportamento de indicadores maternoinfantis e associações com variáveis relacionadas às mães dos recém-nascidos; avaliar os padrões antropométricos dos recém-nascidos, relacionandoos com idade gestacional e comparando-os com estudos dessa mesma natureza realizados em outros locais.

Adicionalmente, a constituição desse banco de dados permitirá a realização de estudos futuros com esse grupo de crianças e suas mães, identificando grupos populacionais de risco para possíveis intervenções.

\section{Métodos}

Esta pesquisa identifica-se como um estudo descritivo, de observação, transversal, acerca das características socioeconômicas, demográficas, comportamentais, reprodutivas, de atenção à saúde materno-infantil, denominado "Estudo Epidemiológico-Social de Saúde Perinatal de Partos Hospitalares da Grande Aracaju".

$\mathrm{O}$ estudo foi planejado tendo como referência a coorte de nascimentos de Ribeirão Preto, 4 iniciada em 1978/1979, sendo importante esse suporte técnico, também, por permitir a realização de comparações entre indicadores de saúde. O banco de dados que se formou viabiliza a realização de subprojetos específicos que estão em andamento.

A grande Aracaju é considerada região metropolitana, composta, segundo a configuração adotada pelo Instituto Brasileiro de Geografia (IBGE), ${ }^{7}$ de quatro municípios: Aracaju, capital do Estado de Sergipe, Barra dos Coqueiros, Nossa Senhora do Socorro e São Cristóvão.

Do ponto de vista econômico, as principais atividades que se destacam em Aracaju são: indústria, comércio, poços petrolíferos concentrados na região litorânea do município, serviços de saúde, sociais e administração pública. O Produto Interno Bruto (PIB) per capita para o ano de 2004 foi de $\mathrm{R} \$ 8.519,00$ (U\$3,276), acima da média do Estado $\mathrm{R} \$ 6.243,00$ (U\$2,401). 8 O Índice de Desenvolvimento Humano (IDH) do município para o ano 2000 era 0,794, similar ao do Brasil no ano de 2003.9

A rede hospitalar de Aracaju é composta por um hospital público de grande porte, três hospitais filantrópicos, um hospital universitário, três clínicas hospitalares privadas, uma maternidade pública, um hospital de cardiologia e duas clínicas psiquiátricas. Todas as unidades hospitalares são conveniadas com o Sistema Único de Saúde (SUS), fazendo-se exceção para uma clínica privada. Nessa rede hospitalar, existem quatro maternidades que atendem a todos os partos nos três níveis de complexidade. Todas dispõem de Unidades de Cuidado Intensivo Neonatal. O maior número de partos $(58,3 \%)$ foi realizado em maternidade conveniada ao SUS, seguidos por uma privada com atendimento ao SUS $(26,0 \%)$, uma pública $(8,2 \%)$ e uma privada não conveniada ao sistema público de saúde (7,5\%).

Durante o ano de 2004, aproximadamente $40 \%$ dos partos de Sergipe ocorreram em Aracaju. Também nos anos anteriores o percentual foi semelhante. Isso vem ocorrendo pelo fato de Aracaju deter o maior número de instalações e leitos hospitalares do Estado. 10

Em Aracaju, a quase totalidade dos nascimentos ocorre em hospitais-maternidade. Em 2004 esse percentual foi de $99,7 \% .10$ A partir dessa constatação foi realizada a previsão do número de nascimentos 
para o ano de 2005, sendo estimado o nascimento de aproximadamente 13.521 crianças, incluindo todos os nascimentos que ocorreriam na grande Aracaju. Optou-se por estudar aproximadamente um terço dos nascidos vivos em 2005, esperando-se uma amostra de 4510 crianças/mães. A estratégia utilizada para este fim foi coletar os dados referentes a todos os nascidos em quatro meses consecutivos, já que não havia sazonalidade na distribuição dos nascimentos e nem de algumas variáveis importantes para o estudo, como as taxas de baixo peso ao nascer, idade materna no parto e gemelaridade. Considerando a previsão da pequena ocorrência de partos (200 nascimentos para todo o ano de 2005) nos hospitais de pequeno porte existentes em São Cristóvão e Nossa Senhora do Socorro, e devido aos custos elevados para a realização da coleta dos dados, não foram coletadas informações nesses municípios.

Fizeram parte do estudo mães que pariram nos quatro hospitais-maternidade de Aracaju procedentes desse município, de Barra dos Coqueiros, Nossa Senhora do Socorro e São Cristóvão. Mães procedentes de outros municípios não foram incluídas. $\mathrm{O}$ acompanhamento dos nascimentos ocorridos em todas as unidades hospitalares foi realizado junto à Secretaria Municipal de Saúde de Aracaju e Secretarias Municipais dos outros municípios inseridos no estudo, por meio do Sistema de Informação de Nascidos Vivos (SINASC). Esta sistemática de trabalho viabilizou a comparação de dados entre os questionários coletados e o SINASC, o que permitiu verificar que o número de nascimentos incluídos era representativo.

Após obtenção da autorização dos diretores dos quatro hospitais, foram selecionados entrevistadores, utilizando-se como critérios possuir experiência anterior em pesquisas epidemiológicas e terem escolaridade mínima correspondente ao ensino médio completo. Todos os entrevistadores foram treinados antes do início do estudo piloto. O treinamento abrangeu uma leitura detalhada de cada pergunta constante no questionário, simulações de entrevistas com os próprios entrevistadores e posteriormente entrevistas nas maternidades com as mães para fazer a aplicação prática do questionário, sempre acompanhados pelos supervisores. Após o treinamento prático foi realizada discussão dos problemas identificados em campo, para padronização na coleta das informações e foram selecionados os entrevistadores que tiveram desempenho considerado satisfatório.

$\mathrm{O}$ estudo piloto foi realizado em duas semanas, ocasião em que foram entrevistadas 513 puérperas dos quatro hospitais-maternidade existentes em Aracaju. A coleta definitiva do estudo iniciou-se em
23 de março de 2005 e foi encerrada em 15 de julho de 2005.

A sistemática de trabalho adotada no estudo consistiu na visita diária pela manhã e à tarde nos quatro hospitais-maternidade do município para verificação da listagem das pacientes internadas em trabalho de parto, objetivando evitar perda de casos. Foi utilizado um livro de registro, em cada unidade hospitalar, para controle dos nascimentos e entrevistas, local onde eram anotadas todas as entrevistas realizadas, dados sobre a data do parto, nome da mãe, sexo, se nascido vivo ou morto. Para a coleta de dados foi utilizado o sistema de entrevistas com as mães, logo após o parto, utilizando-se um questionário construído especialmente para esta finalidade, contendo 114 perguntas abertas e fechadas e dividido em oito blocos: identificação, dados da mãe, dados do pai, dados relativos à gestação, dados referentes ao parto, dados relativos aos recémnascidos, dados do óbito - quando ocorresse, e história obstétrica. Foi elaborado um manual específico para orientação sobre o preenchimento do questionário.

Foram coletados também os dados constantes no prontuário hospitalar da mãe e do recém-nascido e aferidos o peso, comprimento e perímetro craniano dos recém-nascidos. A pesagem considerada foi aquela aferida logo após o nascimento, em balança com sensibilidade de 10 gramas, assim como o perímetro cefálico medido com fita métrica inelástica com precisão de $1 \mathrm{~mm}$. O comprimento dos nativivos, inclusive para prematuros, foi realizado com craveiras padronizadas, também com precisão de 1 mm e especialmente construídas para o estudo. Quando não era possível realizar as medidas na sala de parto, foram consideradas como informações não disponíveis.

Quando não foi possível a realização da entrevista, devido alta hospitalar precoce ou mãe sem condições emocionais de responder ao questionário, foi realizado o preenchimento das fichas, mesmo incompletas, com dados obtidos em registros e prontuários hospitalares. Todas as recusas eram comunicadas aos supervisores e/ou coordenadora de campo que, imediatamente, tentavam revertê-las. Essa estratégia contribuiu para o baixo índice de recusa obtida no estudo $(0,2 \%)$.

Após revisão e discussão semanal, todos os questionários eram revisados durante a coleta dos dados, inicialmente pelos entrevistadores e posteriormente pelos supervisores, que realizavam a conferência das informações e, quando necessário, reencaminhavam aos entrevistadores para correção. Para a codificação foi elaborado um manual específico para 
padronização das informações. Objetivando evitar a perda de casos, foi realizado o acompanhamento dos nascimentos ocorridos em todos os hospitais-maternidade pelos supervisores, que faziam a conferência dos dados e das informações constantes em um questionário sorteado para cumprir essa finalidade.

A idade gestacional foi avaliada pela data da última menstruação (DUM) informada pela mãe. Quando o dia era desconhecido, mas ela lembrava do mês, foi padronizado o dia 15 daquele mês para a realização dos cálculos. Quando ignorado o dia e mês da DUM, utilizou-se o escore de Capurro et al.,11 para cálculo da idade gestacional. Essa avaliação era feita pelo pediatra que recepcionava a criança ao nascer.

O processo de codificação dos questionários foi iniciado após a conclusão da coleta dos dados, realizado por duas entrevistadoras que procediam à codificação do material em espaço apropriado na mesma ficha de entrevista, em campo específico. Concomitantemente, foi realizada a conferência da codificação de cada questionário, por um dos cinco docentes da Universidade Federal de Sergipe envolvido diretamente na pesquisa, em sistema de amostragem (duas de cada dez entrevistas). Inicialmente, foram selecionados 500 questionários para testagem, com revisão da codificação e digitação dupla de $100 \%$ desses questionários. Foi criado arquivo de entrada de dados no programa EpiInfo versão 3.3.2/2005 e foi construída uma máscara para cadastrar todos os questionários. Os questionários foram transcritos para o banco de dados por duas digitadoras de forma independente. O mesmo software também foi utilizado para as análises estatísticas.

$\mathrm{O}$ arquivo de dados foi submetido a diversas verificações para identificar e corrigir inconsistências da codificação e da digitação. Os bancos foram comparados por meio do utilitário Data Compare e foram feitas listagens de dados brutos para conferência e obtidas frequências de variáveis com verificação periódica. Após a correção de cada amostra avaliada, divergências de informações eram discutidas e corrigidas, para que nova testagem então fosse realizada visando identificar erros que pudessem ter sido mantidos. Na primeira testagem, foi verificada margem de erro de $2 \%$, reduzida depois para menos de $1 \%$.

Para preservar as identificações de cunho pessoal das mães dos nativivos, necessária para contatos futuros, objetivando prosseguimento do estudo, foi criado um banco de dados, arquivado separadamente, de forma que todas as informações fossem resguardadas.
Por ocasião do início da pesquisa e durante toda a sua execução, as mídias faladas, escritas e televisadas foram contatadas, objetivando informar e esclarecer toda a população sobre o desenvolvimento e a importância da realização do estudo.

A pesquisa atendeu a todos os princípios éticos requeridos, tendo sido aprovada pelo Comitê de Ética em Pesquisa da Universidade Federal de Sergipe em reunião realizada em 10/12/2004 (Processo n ${ }^{138 / 04) . ~ A ~ p a r t i c i p a c ̧ a ̃ o ~ d a s ~ m u l h e r e s, ~}$ bem como a autorização do responsável, em caso de menores de idade, foi obtida por meio do Termo de Consentimento Livre e Esclarecido. A confiabilidade da informação quanto ao conteúdo da identificação da puérpera, assim como o anonimato por ocasião de futura publicação dos resultados foram assegurados.

Os dados estão apresentados em tabelas constando a frequência e cálculos de percentagens para a maioria dos indicadores investigados (variáveis categóricas) e média, mediana e desvio padrão para as variáveis contínuas (peso, comprimento e perímetro cefálico dos nativivos).

\section{Resultados}

Da amostra de 4806 nativivos obtida nos quatro meses do estudo, foram excluídos $53(1,1 \%)$ gemelares e $7(0,2 \%)$ recusas, restando $4746(98,7 \%)$ recém-nascidos de parto único. Os 74 natimortos do período não fizeram parte do estudo. Dos nascidos vivos participantes da coorte de nascimentos, $5,7 \%$ foram de mães procedentes da área rural. Do total da amostra, 66,6\% eram residentes no município de Aracaju e 58,2 \% oriundos de uma maternidade filantrópica conveniada ao SUS.

Na Tabela 1 estão apresentadas algumas características maternas, renda familiar e escolaridade dos pais. Aproximadamente um quinto das mães $(20,6 \%)$ eram adolescentes. As maiores proporções de partos aconteceram nas faixas etárias de 20 a 24 anos, seguido de 25 a 29 anos $(29,4 \%$ e $24,7 \%$, respectivamente). As mulheres sem companheiros totalizaram $19,6 \%$ da amostra. Apenas 5,3\% das mães fizeram uso do cigarro, enquanto cerca de um quinto $(20,8 \%)$ utilizou bebida alcoólica na gravidez. As primíparas representaram o maior grupo de mães, 39,0\%. Foi verificado também que $7,7 \%$ dos partos ocorreram antes das 37 semanas de gestação (parto pré-termo).

Ainda na Tabela 1 verifica-se que mais da metade das mães vinham de famílias com renda média de um a dois salários mínimos (SM) (56,9\%). O número de mães com renda familiar de menos de um SM foi aproximadamente o quádruplo das que recebiam 10 ou mais salários. Quanto à escolaridade 
Tabela 1

Distribuição dos nativivos de parto único hospitalar segundo características maternas, renda familiar e escolaridade dos pais. Aracaju, 2005.

\begin{tabular}{|c|c|c|}
\hline \multirow[t]{2}{*}{ Características } & \multicolumn{2}{|c|}{ Nascimentos } \\
\hline & $\mathrm{n}$ & $\%$ \\
\hline \multicolumn{3}{|l|}{ Idade materna (anos) } \\
\hline$<20$ & 980 & 20,6 \\
\hline 20 a 24 & 1393 & 29,4 \\
\hline 25 a 29 & 1174 & 24,7 \\
\hline 30 a 34 & 767 & 16,2 \\
\hline$\geq 35$ & 432 & 9,1 \\
\hline \multicolumn{3}{|c|}{ Situação conjugal materna } \\
\hline Sem companheiro & 929 & 19,6 \\
\hline Com companheiro & 3815 & 80,4 \\
\hline \multicolumn{3}{|c|}{ Tabagismo materno na gravidez } \\
\hline Sim & 251 & 5,3 \\
\hline Não & 4493 & 94,7 \\
\hline \multicolumn{3}{|c|}{ Uso de bebida alcoólica pela mãe } \\
\hline Sim & 984 & 20,8 \\
\hline Não & 3760 & 79,2 \\
\hline \multicolumn{3}{|c|}{ Número de doses / semana } \\
\hline$<2$ & 968 & 0,3 \\
\hline$\geq 2$ & 16 & 20,5 \\
\hline Não se aplica & 3760 & 79,2 \\
\hline \multicolumn{3}{|l|}{ Número de gestações } \\
\hline 1 & 1852 & 39,0 \\
\hline 2 & 1204 & 25,4 \\
\hline 3 a 6 & 1513 & 31,9 \\
\hline 7 ou mais & 176 & 3,7 \\
\hline \multicolumn{3}{|c|}{ Duração da gestação (semanas) } \\
\hline$<28$ & 14 & 0,3 \\
\hline 28 a 32 & 47 & 1,0 \\
\hline 33 a 36 & 290 & 6,4 \\
\hline 37 a 41 & 4019 & 89,1 \\
\hline$\geq 42$ & 142 & 3,2 \\
\hline \multicolumn{3}{|c|}{ Renda Familiar (salários mínimos) } \\
\hline$<1$ & 567 & 11,9 \\
\hline 1 a 2 & 2702 & 56,9 \\
\hline 3 a 4 & 814 & 17,2 \\
\hline 5 a 10 & 501 & 10,6 \\
\hline$>10$ & 139 & 2,9 \\
\hline \multicolumn{3}{|c|}{ Escolaridade (anos de estudo) } \\
\hline \multicolumn{3}{|c|}{ Mãe (média = 7,7) } \\
\hline 0 a 4 anos & 909 & 19,2 \\
\hline 5 a 8 anos & 1633 & 34,4 \\
\hline 9 a 11 anos & 1816 & 38,3 \\
\hline$\geq 12$ anos & 354 & 7,5 \\
\hline \multicolumn{3}{|l|}{ Escolaridade } \\
\hline \multicolumn{3}{|l|}{ Pai (média = 7,8) } \\
\hline 0 a 4 anos & 830 & 17,5 \\
\hline 5 a 8 anos & 1352 & 28,5 \\
\hline 9 a 11 anos & 1842 & 38,8 \\
\hline$\geq 12$ anos & 323 & 6,8 \\
\hline prejudicado & 399 & 8,4 \\
\hline
\end{tabular}

Valores prejudicados, todos menores que $5 \%$, não foram apresentados na tabela. 
Tabela 2

Distribuição dos nativivos de parto único hospitalar segundo características da atenção médica e assistência pré-natal e parto. Aracaju, 2005.

\begin{tabular}{|c|c|c|}
\hline \multirow[t]{2}{*}{ Características de atenção médica } & \multicolumn{2}{|c|}{ Nascimentos } \\
\hline & $\mathrm{n}$ & $\%$ \\
\hline \multicolumn{3}{|l|}{ Categoria de pré-natal } \\
\hline Não fez & 73 & 1,5 \\
\hline Sistema Único de Saúde (SUS) & 3606 & 76,0 \\
\hline Particular & 1059 & 22,3 \\
\hline \multicolumn{3}{|l|}{ Número de consultas } \\
\hline não fez & 73 & 1,5 \\
\hline 1 a 3 & 402 & 8,5 \\
\hline 4 a 5 & 1187 & 25,0 \\
\hline$\geq 6$ & 3062 & 64,5 \\
\hline \multicolumn{3}{|l|}{ Profissional que assistiu no pré-natal } \\
\hline Sem Assistência Pré-Natal & 73 & 1,5 \\
\hline Médico do Programa Saúde da Família & 616 & 13,0 \\
\hline Médico e Enfermeiro do Programa Saúde da Família & 2037 & 42,9 \\
\hline Enfermeiro do Programa Saúde da Família & 409 & 8,6 \\
\hline Obstetra & 1606 & 33,9 \\
\hline \multicolumn{3}{|l|}{ Categoria de internação hospitalar } \\
\hline Sistema Único de Saúde (SUS) & 4041 & 85,2 \\
\hline Particular & 695 & 14,6 \\
\hline Prejudicado & 10 & 0,2 \\
\hline \multicolumn{3}{|l|}{ Tipo de parto } \\
\hline Vaginal & 3242 & 68,3 \\
\hline Cesárea & 1498 & 31,6 \\
\hline Fórceps & 5 & 0,1 \\
\hline
\end{tabular}

Valores prejudicados, todos menores que $5 \%$, não foram apresentados na tabela.

dos pais, observa-se um predomínio para ambos da escolaridade de 9 a 11 anos, $38,3 \%$ e $38,8 \%$ respectivamente para as mães e pais dos recém-nascidos. Níveis de escolaridade mais baixos, até oito anos de estudo, predominaram entre as mães $(53,6 \%)$ quando comparadas aos pais (46\%). Contudo, $8,6 \%$ das mães não souberam informar a escolaridade do pai de seu filho. Foi verificada a média de 7,7 anos de estudo para as mães e 7,8 anos para os pais dos nativivos.

A Tabela 2 evidencia algumas características da atenção à assistência pré-natal e ao parto. Quase a totalidade das mães, $98,3 \%$, referiu ter recebido cuidados no pré-natal. Majoritariamente $(76,0 \%)$ esses atendimentos foram realizados no SUS, sendo que cerca de $2 / 3$ tiveram o mínimo de seis consultas $(64,3 \%)$. As mães foram assistidas em pouco mais de 2/3 dos casos por profissionais do Programa Saúde da Família (PSF), 64,5\% (médicos, enfermeiros, médicos e enfermeiros simultaneamente) e apenas
$1 / 3(33,9 \%)$ receberam assistência pré-natal realizada por um obstetra. As pacientes assistidas através do PSF eram, em sua maioria, de baixo risco e quando identificado(s) risco gestacional, eram referenciadas para o nível secundário. Do total dos partos, $85,2 \%$ foram financiados pelo SUS. O parto cesárea representou quase $1 / 3(31,6 \%)$ dos nascimentos.

Foi verificada a predominância do sexo masculino $(51,2 \%)$ na amostra. O baixo peso ao nascer $(<2500 \mathrm{~g})$ apresentou uma frequência maior nos recém-nascidos do sexo feminino $(8,1 \%)$. Os percentuais também foram maiores no sexo feminino para o peso insuficiente ao nascer $(2500 \mid-3000 \mathrm{~g})$ de acordo com a Tabela 3. A média e mediana de peso ao nascer do sexo feminino foi de $3210 \mathrm{~g}$, com desvio padrão de $645,4 \mathrm{~g}$. Para o sexo masculino foi encontrada a média de $3303 \mathrm{~g}$ e mediana de $3302 \mathrm{~g}$, com desvio padrão de $686,5 \mathrm{~g}$. Os recém-nascidos de baixo peso que nasceram pré-termo corresponderam 
Distribuição de algumas características dos nativivos de parto único hospitalar segundo o sexo. Aracaju, 2005

\begin{tabular}{|c|c|c|c|c|c|c|}
\hline \multirow[t]{3}{*}{ Características } & \multicolumn{4}{|c|}{ Nascimentos } & \multicolumn{2}{|c|}{ Total $(\mathrm{N}=4746)$} \\
\hline & \multicolumn{2}{|c|}{$\begin{array}{c}\text { Masculino } \\
(\mathrm{N}=2444)\end{array}$} & \multicolumn{2}{|c|}{$\begin{array}{l}\text { Feminino } \\
(\mathrm{N}=2302)\end{array}$} & & \\
\hline & $\mathrm{n}$ & $\%$ & $\mathrm{n}$ & $\%$ & $\mathrm{n}$ & $\%$ \\
\hline \multicolumn{7}{|c|}{ Peso ao nascer (gramas) } \\
\hline$<1000$ & 17 & 0,7 & 08 & 0,3 & 25 & 0,5 \\
\hline 1.000 a 1.499 & 11 & 0,5 & 11 & 0,5 & 22 & 0,5 \\
\hline 1.500 a 1999 & 25 & 0,5 & 23 & 1,0 & 48 & 1,0 \\
\hline 2.000 a 2.499 & 100 & 4,1 & 145 & 6,3 & 245 & 5,2 \\
\hline 2.500 a 2.999 & 458 & 18,7 & 554 & 24,0 & 1012 & 21,3 \\
\hline 3.000 a 3.499 & 1004 & 41,1 & 945 & 41,1 & 1949 & 41,1 \\
\hline 3.500 a 3.999 & 639 & 26,1 & 495 & 21,5 & 1134 & 23,9 \\
\hline$\geq 4.000$ & 181 & 7,4 & 114 & 5,0 & 295 & 6,2 \\
\hline \multicolumn{7}{|c|}{ Comprimento $(\mathrm{cm})$ ao nascer } \\
\hline$<36$ & 13 & 0,5 & 13 & 0,6 & 26 & 0,5 \\
\hline 36 a 41 & 27 & 1,1 & 21 & 0,9 & 48 & 1,0 \\
\hline 42 a 47 & 514 & 21,0 & 732 & 31,8 & 1246 & 26,3 \\
\hline 48 a 49 & 845 & 34,6 & 809 & 35,1 & 1654 & 34,9 \\
\hline 50 a 51 & 692 & 28,3 & 511 & 22,2 & 1203 & 25,4 \\
\hline$\geq 52$ & 229 & 9,5 & 134 & 5,8 & 363 & 7,7 \\
\hline
\end{tabular}

Valores prejudicados, todos menores que 5\%, não foram apresentados na tabela.

a $48,9 \%(159 / 325)$, enquanto os que nasceram a termo corresponderam a $51,1 \%(166 / 325)$, representando aqueles que possivelmente sofreram restrição do crescimento intrauterino. Ocorreram apenas $0,3 \%$ (16 perdas) de aferição de peso ao nascer, 4,3\% (205) para comprimento ao nascer e 4,9\% (234) para perímetro cefálico.

Cerca de 1/3 dos recém-nascidos do sexo feminino apresentaram comprimento até $47 \mathrm{~cm} \mathrm{(33,3 \% ),}$ enquanto esse valor representou pouco mais de $1 / 5$ dos recém-nascidos do sexo masculino (22,6\%) (Tabela 3). O comprimento dos recém-nascidos apresentou uma média de $51,4 \mathrm{~cm}$ e $50,1 \mathrm{~cm}$ para o sexo masculino e feminino respectivamente, com desvio padrão diferenciado: 1,15 para o sexo masculino e 0,99 para o feminino. Entretanto, a mediana foi 49,0 para ambos os sexos.

Mais de 2/3 das meninas (36,2\%) apresentaram perímetro craniano ao nascer medindo até $34 \mathrm{~cm}$, enquanto essa proporção foi $26,4 \%$ entre os meninos (Tabela 4). A média e a mediana do perímetro craniano foi de $34,5 \mathrm{~cm}$ para os meninos e de $34 \mathrm{~cm}$ para as meninas.

Aracaju apresentou percentuais de baixo peso ao nascer e prematuridade de $7,2 \%$ e $7,7 \%$ respectivamente, com taxa de parto cesária de 31,6\%. A proporção de adequação de pré-natal foi de $64,5 \%$. Foi verificado que $5,3 \%$ das mulheres tinham o hábito de fumar na gestação (Tabela 5). Os dados de outros estudos realizados em Ribeirão Preto, São Luís e Pelotas também se encontram apresentados na Tabela 5. 
Tabela 4

Distribuição das medidas de perímetro craniano dos nativivos de parto único hospitalar segundo o sexo. Aracaju, 2005.

\begin{tabular}{lrrrrrr}
\hline Perímetro craniano & \multicolumn{2}{c}{ Masculino } & \multicolumn{2}{c}{ Feminino } & \multicolumn{2}{c}{ Total } \\
\cline { 2 - 7 } & $\mathrm{n}$ & $\%$ & $\mathrm{n}$ & $\%$ & $\mathrm{n}$ & $\%$ \\
\hline & & & & & & \\
\hline 27 & 8 & 0,3 & 2 & 0,1 & 10 & 0,2 \\
$27-30$ & 14 & 0,6 & 13 & 0,6 & 27 & 0,6 \\
$30-31$ & 26 & 1,1 & 25 & 1,1 & 51 & 1,1 \\
$31-32$ & 58 & 2,5 & 88 & 4,0 & 146 & 3,2 \\
$32-33$ & 151 & 6,5 & 226 & 10,3 & 377 & 8,4 \\
$33-34$ & 357 & 15,4 & 443 & 20,1 & 800 & 17,7 \\
$34-35$ & 556 & 24,0 & 596 & 27,1 & 1152 & 25,5 \\
$35-36$ & 553 & 23,9 & 460 & 20,9 & 1013 & 22,5 \\
$36-37$ & 356 & 15,4 & 227 & 10,3 & 583 & 12,9 \\
$37-38$ & 161 & 7,0 & 81 & 3,7 & 242 & 5,4 \\
$38-39$ & 47 & 2,0 & 26 & 1,2 & 73 & 1,6 \\
$39-40$ & 13 & 0,6 & 6 & 0,3 & 19 & 0,4 \\
$>=40$ & 12 & 0,5 & 7 & 0,3 & 19 & 0,4 \\
Total & 2312 & & 2200 & & 4512 & \\
\hline
\end{tabular}

Ignorado: sexo masculino $=132(5,4 \%)$; sexo feminino $=102(4,4 \%)$.

Tabela 5

Características da população estudada e de algumas coortes brasileiras de nascimento.

\begin{tabular}{|c|c|c|c|c|c|c|}
\hline \multirow[t]{2}{*}{ Local/Estudo } & $\begin{array}{c}\text { Baixo Peso } \\
\text { ao nascer }\end{array}$ & Pré-termo & $\begin{array}{c}\text { Parto } \\
\text { cesárea }\end{array}$ & $\begin{array}{l}\text { Pré-natal } \\
\text { adequado }\end{array}$ & $\begin{array}{l}\text { Fumo na } \\
\text { gestação }\end{array}$ & $<35 M$ \\
\hline & $\%$ & $\%$ & $\%$ & $\%$ & $\%$ & $\%$ \\
\hline Aracaju, 2005.18 & 7,2 & 7,7 & 31,6 & 64,5 & 5,3 & 68,8 \\
\hline Ribeirão Preto, 1978/1979.4 & 7,2 & 7,6 & 30,3 & $76,6 \mathrm{~b}$ & 28,9 & $45,1 d$ \\
\hline \multirow[t]{2}{*}{ Ribeirão Preto, 1994.2} & 10,6 & 13,6 & 50,9 & $91,0^{b}$ & 17,0 & $31,9 d$ \\
\hline & & & & $71,4 a$ & & $22,3 c$ \\
\hline São Luis, 1997/1998. 5 & 7,6 & 12,7 & 33,7 & 51,4 & 5,7 & 61,7 \\
\hline Pelotas, 1982.13 & 9,0 & 5,6 & 27,7 & 80,3 & 35,6 & 69,5 \\
\hline Pelotas, 1993.2 & 9,8 & 7,5 & 30,5 & 86,2 & 33,4 & 60,8 \\
\hline Pelotas, 2004.2 & 10,0 & 15,3 & 45,4 & 82.5 & 27,5 & 66,9 \\
\hline
\end{tabular}

a Conforme considerado no respectivo estudo; a $\geq 6$ consultas, inicio até 120 dias, ajustadas a idade gestacional; b $\geq 4$ consultas; $S M=$ Salário mínimo; $c \geq 3$ SM; $d \geq 4$ SM.

\section{Discussão}

Aracaju é local bastante adequado à realização de estudos da natureza que está sendo apresentado aqui, em vista do tamanho da cidade propiciar pesquisas de base populacional, bem como pelo alto número de nascimentos que ocorre no ambiente hospitalar.
Além disso, as mães que participaram de forma efetiva desse estudo entenderam a sua importância, o que foi demonstrado pelo número muito baixo de recusas.

Este é o primeiro estudo epidemiológico-social da saúde materno-infantil de partos hospitalares realizado no Estado de Sergipe. Estudos dessa magni- 
tude são importantes, pois têm um papel fundamental na realização de pesquisas de base populacional, haja vista que a maioria dos estudos é realizada com dados secundários, muitas vezes, subnotificados e com restrição de variáveis a serem estudadas. Pelo fato de ter sido utilizada metodologia semelhante em relação à população de nascidos vivos, é adequada a comparação com alguns dados de estudos realizados na cidade de Ribeirão Preto, São Luís e Pelotas. Ribeirão Preto, com Índice de Desenvolvimento Humano (IDH) de 0,855 em 2000, ${ }^{8}$ localizada na mais rica região do interior de São Paulo, na região Sudeste do país, tem duas coortes em seguimento (1978-79 e 1994), sendo a primeira a mais antiga do país. Pelotas (IDH 0,816 em 2000), 8 município do Rio Grande do Sul (região Sul), tem três coortes sob seguimento (1982, 1993 e 2004). São Luís (IDH 0,778 em 2000), 8 capital do Maranhão, no Nordeste, tem a coorte de 1997-98 em acompanhamento.

Esta pesquisa evidencia que os hospitais-maternidade conveniados ao SUS são os responsáveis pelo maior número de partos, da mesma forma que em outros locais do país.12,13 Esse fato ratifica os dados oficiais que apontam $86 \%$ da população do município de Aracaju 12 como SUS-dependente. Na coorte de São Luís, em 1997/1998,5 mostrou-se que $90 \%$ dos partos foram financiados pelo SUS.

Mais da metade das mães era de famílias com renda de um a dois salários, sendo que em São Luís esse percentual foi de pouco mais de um terço $(35,5 \%), 5$ semelhante ao encontrado em Pelotas nas três coortes.2,13 Quando comparada a renda familiar acima de 10 SM, observa-se que em Ribeirão Preto em 19942 essa faixa de renda era aproximadamente quatro vezes maior $(11,4 \%$ contra $2,8 \%)$.

Foi observado que a proporção de mães adolescentes neste estudo foi mais baixa que a encontrada em São Luís $(29,4 \%)^{14}$ e mais elevada que a de Pelotas em 20042 (18,9\%). Aproximadamente um quinto das mães informaram não possuir companheiro, percentual inferior ao encontrado em São Luís $(22,6 \%)^{5}$ e quase duas vezes maior que a verificada em Ribeirão Preto em 19911 (11,4\%). A prematuridade $(7,7 \%)$ foi muito menor do que a verificada em Ribeirão Preto em 1994 e em São Luis em 1997-98 (14,8\% e 12,4\%, respectivamente), 5 e comparável à de Pelotas 12 anos antes. Convém ressaltar que tanto em Ribeirão Preto como em Pelotas houve aumento progressivo do percentual de baixo peso ao nascer e de parto prematuro no decorrer do tempo.

Comportamento semelhante aconteceu no que se refere ao tabagismo na gestação, que apresentou a proporção de 5,3\% de gestantes fumantes em Aracaju, contra 5,7\% em São Luís. 5 Em Ribeirão Preto esse percentual foi de $28,9 \%$ em 1978/79 e em Pelotas, 35,6\% em 1982.4,13 Nessas cidades houve queda progressiva no tabagismo materno durante a gestação. A proporção de mães que referiu ter feito uso de bebida alcoólica na gravidez foi elevada $(20,8 \%)$, diferentemente da verificada em Pelotas em $2004(3,3 \%) .^{2}$ No entanto não foram investigadas a quantidade e frequência do uso.

Em relação à paridade, os dados encontrados no presente estudo são semelhantes aos da coorte de 1994 realizada na cidade de Ribeirão Preto, ${ }^{1}$ onde $36,8 \%$ eram primigestas e aos de Pelotas (2004) $(39,4 \%) .{ }^{3}$ No entanto, em São Luís (1997/98), esse percentual era bem maior $(47,8 \%) .5$

A assistência pré-natal tem papel relevante na saúde materna e do recém-nascido e pode ser vista como política compensatória, cabendo-lhe o papel de minimizar o efeito das desigualdades socioeconômicas. 15 Nesse estudo, apenas 1,5\% das mães referiu não ter sido assistida durante o pré-natal, número inferior aos encontrados em Recife, 6,3\%,16 São Luís, 11,5\%,15 Pelotas, 5,0\%17 e semelhante ao observado em Ribeirão Preto, 2,4\%. ${ }^{1}$ Outro dado de destaque foi referente aos profissionais que assistiram as mães durante o pré-natal. Profissionais do PSF foram responsáveis por $64,5 \%$ dos atendimentos. Esse percentual possivelmente se justifica por Sergipe ter uma cobertura do PSF de 87,3\%, uma das mais elevadas do Brasil,18 bem como por Aracaju ter sido selecionada pelo Ministério da Saúde como uma das cidades piloto para implantação do PSF e do cartão SUS.

$\mathrm{Na}$ assistência ao parto prevaleceu a categoria de internação SUS, similar aos estudos realizados em São Luís, todavia, com proporções mais baixas. ${ }^{5} \mathrm{~A}$ proporção de parto cesárea encontrada nesse estudo, embora elevado para os parâmetros recomendados (10 a $15 \%$ ) foi de $31,6 \%$, inferior à de outras coortes. 19,20 Também o percentual de baixo peso ao nascer na grande Aracaju foi menor $(7,1 \%)$ que os das cidades de Ribeirão Preto $(12,4 \%)$, Pelotas $(10,0 \%)$ e São Luís $(9,6) .1,2,5$

A análise dos diversos resultados e comparações aqui apresentados parece confirmar a existência de um paradoxo entre os dados das coortes de Ribeirão Preto e São Luís, ${ }^{21}$ aqui reforçados pela semelhança com os dados de Aracaju e Pelotas. Assim é que Aracaju e São Luís são cidades com menor poder aquisitivo e disponibilidade de serviços de saúde e possuem menores percentuais de baixo peso ao nascer e parto prematuro que as cidades da regiões mais desenvolvidas do país. Aracaju tem um 
percentual muito baixo de mães fumantes, só comparável a São Luís, mas ainda assim menor. Também o percentual de parto cesárea foi o menor das quatro cidades, se considerarmos os últimos estudos de cada uma.

Somente o estudo de Ribeirão Preto em 1978/1979 e o presente estudo avaliaram o perímetro craniano dos recém-nascidos. As médias do perímetro cefálico (PC), em Aracaju, são muito próximas das médias observadas no estudo de Ribeirão Preto 22 para os dois sexos. As médias do PC também foram muito semelhantes às de recémnascidos da Grande Beirute, no Líbano, 23 entre 1999 e $2001(34,58 \mathrm{~cm}$ para meninos e $33,13 \mathrm{~cm}$ para meninas), e cerca de $0,5 \mathrm{~cm}$ menor do que das crianças suecas $(35 \mathrm{~cm}$ e $34,5 \mathrm{~cm}$ para meninos e meninas, respectivamente). Em estudo que observou a variação geográfica no tamanho e proporções corporais de recém-nascidos, comparando-se medidas antropométricas coletadas de 20 estudos envolvendo países de diferentes níveis de desenvolvimento, 24 esses autores observaram que a variação no PC foi de 2,3\%. Essa variação se reduziu para $1,3 \%$ com a exclusão dos dados de Pequim, China, cuja média geral foi de $32 \mathrm{~cm}$ e desvio-padrão de 1,5 $\mathrm{cm}$. Essa variação geográfica tem múltiplas razões, que inclui efeitos de genes herdados (selecionados pelas condições ambientais do passado), que controlam hormônios fetais do crescimento, e efeitos do ambiente fetal atual (a mãe), que controla o suprimento de nutrientes para o feto.

Os dados obtidos neste estudo permitem situar Aracaju em percentuais de baixo peso ao nascer, prematuridade e parto cesárea semelhantes a Ribeirão Preto em 1978/79; relativo ao parto cesárea, os resultados foram equivalentes a Pelotas em 1993. A adequação do pré-natal somente foi maior que a de São Luís em 1997/98, enquanto o tabagismo na gestação foi o menor entre as coortes analisadas.

$\mathrm{Na}$ realização de um estudo epidemiológico desse porte, muitos foram os desafios e obstáculos que necessitaram ser superados. Uma das maiores dificuldades foi a necessidade de vigilância constante para evitar perda de dados e o acompanhamento diário dos entrevistadores, objetivando a uniformidade das informações. Ocorreram também alguns problemas técnicos, em vista deste ser o primeiro trabalho dessa natureza realizado no estado, entretanto, todos foram resolvidos e contribuíram para o crescimento qualitativo do estudo.

O presente estudo demonstra ser possível a realização de pesquisas de investigação de cunho sociodemográfico, de base populacional em nosso meio. É relevante, novamente, destacar que essa pesquisa constituirá um banco de dados que permitirá a realização de estudos prospectivos, com seus resultados podendo ser utilizados para o planejamento das ações de saúde direcionadas ao grupo materno-infantil.

\section{Agradecimentos}

Somos agradecidos às direções de todos os hospitais e a todos os funcionários pela atenção, colaboração e receptividade. Aos supervisores, entrevistadores e digitadores nossos agradecimentos pelo compromisso e responsabilidade na condução de todas as etapas do estudo. Expressamos nosso agradecimento ao Conselho Nacional de Desenvolvimento Científico e Tecnológico (CNPq), pelo financiamento deste Estudo (Processo n. ${ }^{\circ}$ 620110/04). Nossa gratidão especial às mães e seus recém-nascidos pela cooperação e, principalmente, por acreditarem na importância do estudo.

\section{Referências}

1. Bettiol H, Barbieri MA, Gomes UA, Andrea M, Goldani MZ, Ribeiro ER. Saúde perinatal: metodologia e características da população estudada. Rev Saúde Pública. 1998; 32: 1828.

2. Barros AJ, da Silva dos Santos I, Victora CG, Albernaz, EP, Domingues, MR, Timm, IK, Matijasevich, A, Bertoldi, AD, Barros, CF. A coorte de nascimentos de Pelotas-2004: métodos e descrição. Rev Saúde Pública. 2006; 40: 402-13.
3. Batty GD, Alves JG, Correia J, Lawlor DA. Examining lifecourse influences on chronic disease: the importance of birth cohort studies from low- and middle- income countries. An overview. Braz J Med Biol Res. 2007; 40: 127786.

4. Barbieri MA, Gomes UA, Barros Filho AA, Bettiol H, Almeida LCA, Silva AAM. Saúde perinatal em Ribeirão Preto, SP, Brasil: a questão do método. Cad Saúde Pública. 1989; 5: 376-87. 
5. Silva AAM, Coimbra L, Silva RA, Alves MTSSB, Lamy Filho F. Perinatal health and mother-child health care in the municipality of São Luís, Maranhão, Brasil. Cad Saúde Pública. 2001; 17: 1413-23.

6. Victora CG, Barros FC, Halpern R, Menezes AMB, Horta BL, Tomasi E. Estudo longitudinal da população maternoInfantil na região urbana do sul do Brasil, 1993: aspectos metodológicos e resultados preliminares. Rev Saúde Pública. 1999; 30: 34-45.

7. IBGE (Instituto Brasileiro de Geografia e Estatística) Síntese dos indicadores sociais. Estudos e pesquisa de informações demográficas e socioeconômicas. Rio de Janeiro; 2000.

8. IBGE (Instituto Brasileiro de Geografia e Estatística). Produtos Internos Brutos a preços correntes e per capita nas unidades da federação e municípios. 2001-2004. Rio de Janeiro; 2007.

9. IBGE (Instituto Brasileiro de Geografia e Estatística). Perfil dos municípios brasileiros 2003. Rio de Janeiro; 2007.

10. Sergipe. Governo. Situação de saúde de Sergipe: 2004. Aracaju; 2005.

11. Capurro H, Konichezky S, Fonseca D, Caldeyro-Barcia R. A simplified method for diagnosis of gestational age in the newborn infant. J Pediatr. (Rio de J.) 1978; 93: 120-2.

12. Ribeiro ER, Barbieri MA, Bettiol H, Silva AA. Comparação entre duas coortes de mães adolescentes em município do Sudeste do Brasil. Rev Saúde Pública. 2000; 34: 136-42.

13. Victora CG, Barros FC, Martines JC, Beria JU, Vaughan JP. Estudo longitudinal das crianças nascidas em Pelotas em 1982. Metodologia e resultados preliminares. Rev Saúde Pública. 1985; 19: 58-68.

14. Simões VMF, Silva AAM, Bettiol H, Lamy Filho F, Tonial SR, Gomide ME. Características da gravidez na adolescência em São Luis, Maranhão. Rev Saúde Pública. 2003; 39: 559-65.

16. Pereira MHP, Frias PG, Carvalho IC, Vidal AS, Figueiroa JN. Mortalidade neonatal hospitalar na coorte de nascidos vivos em maternidade-escola na Região Nordeste do Brasil 2001-2003. Epidemiol Serv Saúde. 2007; 15: 9-28.

Recebido em 4 de janeiro de 2008

Versão final apresentada em 28 de outubro de 2008

Aprovado em 20 de novembro de 2008
15. Alves MTSSB, Silva AAM. Avaliação de qualidade de maternidade: assistência à mulher e ao recém-nascido no SUS. São Luís: UNICEF; 2000.

17. Halpern R, Barros FC, Victora CG, Tomasi E. Atenção prénatal em Pelotas, Rio Grande do Sul, Brazil, 1993. Cad Saúde Pública. 1998; 14: 487-92.

18. Sergipe. Secretaria de Saúde. Gestão Estadual do SUS: 2003-2006. Aracaju; 2006.

19. Victora CG, Barros FC, Lima RC, Behague DP, Gonçalves $\mathrm{H}$, Horta BL. The Pelotas birth cohort study, Rio Grande do Sul, Brazil, 1982-2001. Cad Saúde Pública. 2003; 19: 124156.

20. Goldani MZ, Bettiol H, Barbieri MA, Tomkins A. Maternal age, social changes, and pregnancy outcome in Ribeirao Preto, southeast Brazil, in 1978-79 and 1994. Cad Saúde Pública. 2000; 16: 1041-7.

21. Silva AA, Bettiol H, Barbieri MA, Brito, LGO, Pereira, MM, Aragão, VMR, Ribeiro, VS. Which factors could explain the low birth weight paradox? Rev Saúde Pública. 2006; 40: 648-

22. Gomes UA, Rosado ML, Moura-Ribeiro MV, Ricco RG, Bettiol H, Barbieri MA. Saúde perinatal em Ribeirao Preto, Brasil: distribuição do perímetro cranaiano em recémnascidos. Arq Neuropsiquiatr. 1989; 47: 392-6.

23. Khalid A, Yunis KA, Khawaja M, Beydoun H, Nassif Y, khogali M, Tamim, H. Intrauterine growth standards in a developing country: a study of singleton livebirths at 28-42 weeks' gestation. Paediatr Perinatal Epidemiol. 2007; 21: 387-96.

24. Leary S, Fall C, Osmond C, Lovel H, Campbell D, Eriksson J. Geographical variation in neonatal phenotype. Acta Obstet Gynecol Scand. 2006; 85: 1080-9. 